\title{
A path-integral approach to the problem of time
}

\author{
M. M. Amara荬 and Martin Bojowald \\ Institute for Gravitation and the Cosmos, \\ The Pennsylvania State University, \\ 104 Davey Lab, University Park, PA 16802, USA
}

\begin{abstract}
Quantum transition amplitudes are formulated for a model system with local internal time, using path integrals. The amplitudes are shown to be more regular near a turning point of internal time than could be expected based on existing canonical treatments. In particular, a successful transition through a turning point is provided in the model system, together with a new definition of such a transition in general terms. Some of the results rely on a fruitful relation between the problem of time and general Gribov problems.
\end{abstract}

\section{Introduction}

In relativistic systems, the lack of an absolute time parameter makes it impossible to implement the usual requirement of unitary quantum evolution in a straightforward manner. A local time variable is valid only for a finite range, so that coordinate changes are required to patch together classical trajectories. Corresponding transformations between quantum theories, describing the same system but based on different time choices, should then be used in order to patch together piecewise quantum evolutions. However, if evolution is unitary in each patch, it extends beyond the classical range of the time parameter. The physical meaning of such an extension is unclear, in particular if it happens in semiclassical regimes such as small curvature of a cosmological model. Transformations between different time choices, even global ones, are important also to guarantee covariance of the quantum theory. Since such transformations have not been defined to complete satisfaction, the problem of time [1, 2, 3] remains open. (See [4, 5, 6] for recent ideas on transformations of time choices.) But before one can introduce such transformations between different local internal times, the local quantum evolution itself must be made well-defined, generalizing the usual unitary evolution operators. An important question is then the behavior of evolution near a turning point of local internal time, close to where it ceases to be a good time variable.

Here, we begin a new investigation based on the relationship between time choices and gauge fixings, both of which may be globally valid in simple examples but in general hold only locally. The relationship between time and gauge is easy to see because the absence

*e-mail address: mramaciel@gmail.com

${ }^{\dagger}$ e-mail address: bojowald@gravity.psu.edu 
of an absolute time in relativistic systems is formally realized by having a Hamiltonian constraint, replacing the usual Hamiltonian which can take different non-zero energy values. Classically, the Hamiltonian constraint $C$ plays a dual role. It generates evolution with respect to a fixed time choice by Hamiltonian equations $\dot{f}=\{f, N C\}$, where different choices of the phase-space function $N$ correspond to evolution with respect to different time coordinates. The Hamiltonian constraint also generates transformations between different time choices by the gauge transformations $\mathrm{d} f / \mathrm{d} \epsilon=\{f, C\}$. A convenient way of fixing time choices, following Dirac [7], is to use an internal time, or to select one of the phase-space variables, called $\phi$ in what follows, as the time parameter $\tau$. For $\phi$ to be able to play the role of time at least locally, it must be non-constant, so that $\dot{\phi}=\{\phi, N C\} \neq 0$ in some part of phase space. The time choice, expressed for instance as $G:=\phi-\tau=0$, then amounts to a (local) gauge fixing of the Hamiltonian constraint.

The internal time as well as the gauge fixing are global if $\{\phi, C\}=\{G, C\}$ does not become zero as $\tau$ changes on the family of gauge-fixed hypersurfaces $C=0=G$. This condition is easily seen to be realized in simple cases in which $C$ depends on the momentum $p_{\phi}$ of $\phi$ via a standard kinetic term, but not on $\phi$ itself. We then have $\{\phi, C\} \propto p_{\phi}$ and $\dot{p}_{\phi}=\left\{p_{\phi}, C\right\}=0$. However, most realistic field models have interaction potentials for all phase-space variables, so that $\phi$-dependent potentials contribute to the Hamiltonian constraint for all possible choices of $\phi$. In such cases, global internal times do not exist, and gauge fixings of the form $\phi-\tau=0$ are subject to Gribov problems [8]. (For a review of Gribov problems in gauge theories, see for instance [9].)

Quantization in the presence of Gribov problems can be dealt with conveniently if one uses path integrals. In the next section, we will therefore begin our discussion by a brief review of these methods, making contact with previous work on path-integral quantizations of free relativistic particles [10, 11]. Our new results are related to an extension of these earlier constructions to model systems with time-dependent potentials, which require local internal times. We will focus here on non-technical issues in order to highlight interesting features in this new situation. In Section 3, we will suggest a possible definition of evolution across turning points of local internal times, but also point out open questions regarding a complete realization of this scheme. However, in spite of these difficulties, the path-integral treatment is shown to have some advantages compared with alternative approaches: it appears less singular close to turning points than canonical descriptions which make use of kinematical structures in intermediate derivations.

Local internal times have been implemented semiclassically in canonical language, using methods of effective constraints [12, 13, 14]. A comparison with path-integral results is instructive and is provided in Section 4. In particular, the canonical framework distinguishes between kinematical variables, some of which take complex values, and physical ones on which reality conditions are imposed. Path-integral versions show only the latter properties, which leads to technical simplifications but also makes it more difficult to see some of the underlying features, in particular those related to transformations of time choices. Nevertheless, the successful evolution through a turning point, provided here in a simple model system, is promising and suggests new questions to be analyzed in the context of the problem of time. 


\section{The problem of time as a Gribov problem}

We consider systems with two canonical pairs of degrees of freedom, $(q, p)$ and $\left(\phi, p_{\phi}\right)$, subject to one constraint $C=0$ which for now will be assumed to be of the form $C=$ $p_{\phi}^{2}-H(q, p, \phi)^{2}$. (We will later specify $H(q, p, \phi)$ in more detail.) Such systems have an action principle

$$
S=\int_{\tau_{\mathrm{a}}}^{\tau_{\mathrm{b}}} d \tau\left(p \dot{q}+p_{\phi} \dot{\phi}-N C\right)
$$

where $N$ is an auxiliary variable with zero momentum, $p_{N}=0$. Varying by $N$ imposes the constraint $C=0$.

Choosing $\phi$ as an internal time is equivalent to choosing the gauge fixing $G=\phi-f(\tau)=$ 0 with a linear function $f(\tau)$. For now, we choose $f(\tau)=\tau$. In order for this formulation to be meaningful, we have to introduce a family of copies of the original phase space, labeled by the parameter $\tau$. On each copy, $G=0$ sets $\phi$ to a constant value $\tau$. The gauge fixing

is global only if the gauge flow generated by the constraint $C$ on $\phi, \delta \phi / \delta \epsilon=\{\phi, C\}$, has solutions monotonic in $\epsilon$. Since the same equation can, in the language of canonical general relativity, be interpreted as proper-time evolution of $\phi$, a global gauge fixing is obtained if $\phi(\tau)$ evolves without turning points. With our choice of $C$, turning points are reached whenever the trajectory crosses the hyperplane $p_{\phi}=0$ in phase space.

One can see the relationship with $p_{\phi}=0$ in a more formal way by considering the path integral of our gauge-fixed system. Combining the constraint $C=0$ with the gauge-fixing condition $G=0$ turns our system into one with a pair of second-class constraints. The constraint surface where $C=0$ and $G=0$ then has a symplectic structure given by Dirac brackets instead of the original Poisson brackets of canonical variables. Correspondingly, the path-integral measure must be modified by a factor which turns out to be equal to the Faddeev-Popov determinant [15, 16], or a simple Faddeev-Popov function $|\{C, G\}|$ in the case of a single pair of constraints. For our general form of $C$ and the chosen gauge fixing, $|\{C, G\}|=2\left|p_{\phi}\right|$ which vanishes just at the turning points of $\phi$. The path-integral measure is degenerate where $p_{\phi}=0$.

If $p_{\phi}=0$ is reached on a gauge orbit, the gauge fixing is not global but subject to a Gribov problem. The hypersurface in phase space where $p_{\phi}=0$ is called a Gribov horizon, separating regions in which the gauge fixing may be used locally. In order to avoid overcounting degrees of freedom, the path integration should be restricted to one of the Gribov regions, in this case $p_{\phi}>0$ or $p_{\phi}<0$. This restriction can easily be performed by inserting a step function $\theta\left( \pm p_{\phi}\right)$ in the path integral. We arrive at the path-integral expression

$$
\left(q_{\mathrm{a}} \phi_{\mathrm{a}} \tau_{\mathrm{a}} \mid q_{\mathrm{b}} \phi_{\mathrm{b}} \tau_{\mathrm{b}}\right)=\int D q D p D \phi D p_{\phi} D N \cdot 2\left|p_{\phi}\right| \theta\left( \pm p_{\phi}\right) \delta(G) \exp \left(\frac{i}{\hbar} \int_{\tau_{\mathrm{a}}}^{\tau_{\mathrm{b}}} d \tau\left(p \dot{q}+p_{\phi} \dot{\phi}-N C\right)\right)
$$

for transition amplitudes, with paths restricted so that $q\left(\tau_{\mathrm{a}}\right)=q_{\mathrm{a}}, q\left(\tau_{\mathrm{b}}\right)=q_{\mathrm{b}}, \phi\left(\tau_{\mathrm{a}}\right)=\phi_{\mathrm{a}}$ and $\phi\left(\tau_{\mathrm{b}}\right)=\phi_{\mathrm{b}}$. 
While the relationship between a measure factor of $2 p_{\phi}$ in path-integral treatments of the free relativistic particle and the Faddeev-Popov factor has been recognized in [10], the step function had in this paper been inserted so as to restrict to positive frequencies, stating that it has no counterpart in gauge theories. For a free particle, the relationship is indeed not obvious because it has a constant $p_{\phi}$ on gauge trajectories, so that the hypersurface $p_{\phi}=0$ is never crossed and does not constitute a Gribov horizon. The language of Gribov problems in the context of internal times is therefore meaningful only if the internal time used is local and has a turning point. (A possible relationship between the Gribov problem and step functions in path integrals for a free relativistic particle has been conjectured but not pursued in [11].)

Choosing the negative sign of $p_{\phi}$, to be specific, we can solve the constraint by $p_{\phi}=$ $-|H(q, p, \phi)|$. This classical Hamiltonian also appears in a path-integral version of (2) in which integrations over $N, p_{\phi}$ and $\phi$ have been carried out explicitly: Integration over $N$ in (2) leads to a delta function

$$
\delta(C)=\delta\left(p_{\phi}^{2}-H^{2}\right)=\frac{1}{2\left|p_{\phi}\right|}\left(\delta\left(p_{\phi}-|H|\right)+\delta\left(p_{\phi}+|H|\right)\right) .
$$

The factor of $\left(2\left|p_{\phi}\right|\right)^{-1}$ cancels out with the Faddeev-Popov function, while the first delta function is removed by the step function restricting integrations to the Gribov region with negative $p_{\phi}$. A single delta function is then left, which trivializes the integration over $p_{\phi}$. The $\phi$-integration is trivialized by $\delta(G)$ for the gauge fixing, setting $\phi=\tau$. Performing these integrations, we are left with a standard path integral

$$
\left(q_{\mathrm{b}} \tau_{\mathrm{b}} \mid q_{\mathrm{a}} \tau_{\mathrm{a}}\right)=\int D q D p \exp \left(i \hbar^{-1} \int_{\tau_{\mathrm{a}}}^{\tau_{\mathrm{b}}} \mathrm{d} \tau(p \dot{q}-|H(q, p, \tau)|)\right)
$$

with a time-dependent Hamiltonian. At this stage, $\phi$ and $\tau$ are no longer independent.

As long as no turning point of $\phi$ is crossed between $\tau_{\mathrm{a}}$ and $\tau_{\mathrm{b}}$, this standard path integral can be used for our original system with local internal time $\phi$. Evolution across a turning point is more complicated. It requires us to change the branch from negative $\mathrm{d} \phi / \mathrm{d} \tau$ to positive $\mathrm{d} \phi / \mathrm{d} \tau$. We could use our path integral written above to evolve all the way up to a turning point at $\tau_{\mathrm{t}}$, continued with an analogous expression for the other branch to go from $\tau_{\mathrm{t}}$ to the final $\tau_{\mathrm{b}}$. These two branches could be connected by the usual composition rule

$$
\left(q_{\mathrm{b}} \tau_{\mathrm{b}} \mid q_{\mathrm{a}} \tau_{\mathrm{a}}\right)=\int \mathrm{d} q_{\mathrm{t}}\left(q_{\mathrm{b}} \tau_{\mathrm{b}} \mid q_{\mathrm{t}} \tau_{\mathrm{t}}\right)_{+}\left(q_{\mathrm{t}} \tau_{\mathrm{t}} \mid q_{\mathrm{a}} \tau_{\mathrm{a}}\right)_{-}
$$

where the \pm -signs indicate the branch used. At a turning point, the original phase-space structure is ill-defined because the Dirac brackets on the hypersurface $C=0=G$ are infinite, or the path-integral measure is degenerate. However, after gauge fixing the path integrals in both branches, $\phi$ and $p_{\phi}$ have been integrated out, so that the degeneracy of their measure no longer plays a role.

Provided that the path integrals in both branches can be computed with a timedependent Hamiltonian $|H|$ near the turning point where $H=0$, evolution through the 
turning point can be defined. A problem may arise at this stage because $|H|$ is not regular at $H=0$ in many cases of interest. For a time-dependent constraint in relativistic systems, for instance, the Hamiltonian in (4) may be of the form $H=\sqrt{p^{2}+m^{2}-V(\tau)}$ with a root-like pole at the turning point. Far from the turning point the Hamiltonian can be approximated by using the "non-relativistic" expansion $H=m+\left(p^{2}-V(\tau)\right) / 2 m+\cdots$, leading to standard path integrals. But close to the turning point the square root cannot be expanded. These questions will be shown to be solvable in a simple example discussed in the next section. The main question, however, is how to implement a gauge fixing that takes into account the fact that $\phi$ "runs backwards" after it reaches the turning point. We will provide more details in the specific model of the next section.

\section{$3 \quad$ Evolution through a turning point}

The standard treatment of a global internal time $\phi$, as used in the preceding section, makes use of a clear distinction between the two possible signs of $p_{\phi}$. One of them, usually the negative sign so as to match up with the common time dependence $\exp (-i E t / \hbar)$ of stationary states in quantum mechanics, is taken as the one governing evolution forward in time, while the opposite sign corresponds to backward evolution. If $\phi$ is a local internal time with turning points, the clear distinction disappears because on a single gauge orbit, $p_{\phi}$ changes sign. The variable to be taken as internal time moves forward and backward on the same trajectory $\phi(\epsilon)$, but it should also be possible to consider the trajectory in reverse. There are now at least four different regimes - forward and backward evolution before as well as after the turning point - and a choice with only two options, such as the sign of $p_{\phi}$, is no longer sufficient to distinguish all of them from one another.

In general, disentangling forward and backward evolution along trajectories can be rather cumbersome, in particular when a local internal time with several possible turning points is considered. We will therefore specialize our model system further, so that we have a local internal time $\phi$ with a single turning point. This feature can be achieved by choosing a linear $\phi$-dependent potential, for which the constraint is

$$
C=p_{\phi}^{2}-p^{2}-m^{2}+\lambda \phi .
$$

Here, we also specialized the dependence on the evolving pair $(q, p)$. In particular, there is no $q$-dependent potential (so that one could use $q$ as a global internal time in this model) and $p$ is conserved. These properties will imply further simplifications in the detailed construction. At the end of our analysis we will briefly comment on more general models.

Before we proceed, we note that turning points and Gribov horizons can be caused not only by potentials depending on internal time but also by topological effects. An example for such topological turning points has recently been discussed in detail in [17]. For instance, if the configuration space of one coordinate $\phi$ is a circle, globally defined basic variables are $\cos \phi$ and $\sin \phi$ together with $p_{\phi}$. A simple-looking, $\phi$-independent constraint such as $C=p_{\phi}^{2}-p^{2}$ would then lead to Gribov horizons on the non-trivial phase space because $\{\cos \phi, C\}=-2 p_{\phi} \sin \phi$ becomes zero even if the constant $p_{\phi}$ is non-zero. However, 
such models and their turning points are different from those studied here because they do not lead to the difficulty of assigning signs of $p_{\phi}$ to different branches of orbits before and after the turning point.

\subsection{Classical formulation}

While $\phi$ has a turning point at

$$
\phi_{\mathrm{t}}=\frac{p^{2}+m^{2}}{\lambda}
$$

$q$ is monotonic with respect to the gauge parameter $\epsilon$. We have

$$
\frac{\mathrm{d} q}{\mathrm{~d} \epsilon}=\{q, C\}=-2 p
$$

and therefore $q(\epsilon)=q_{0}-2 p \epsilon$ where $p$ is constant. In terms of local $\phi$-evolution, we have

$$
\frac{\mathrm{d} q}{\mathrm{~d} \phi}=\left(\frac{\mathrm{d} \phi}{\mathrm{d} \epsilon}\right)^{-1} \frac{\mathrm{d} q}{\mathrm{~d} \epsilon}=-\frac{p}{p_{\phi}} \approx \pm \frac{p}{\sqrt{p^{2}+m^{2}-\lambda \phi}}
$$

for $p_{\phi}=\mp \sqrt{p^{2}+m^{2}-\lambda \phi}$. The solution is

$$
q(\phi)=q_{0} \mp \frac{2}{\lambda} p\left(\sqrt{p^{2}+m^{2}-\lambda \phi}-\sqrt{p^{2}+m^{2}-\lambda \phi_{0}}\right)
$$

as long as $\phi$ and $\phi_{0}$ are connected by evolution that does not go through the turning point of $\phi$. For $\phi$ moving toward the turning point at $\phi_{\mathrm{t}}$, it is increasing so that $\left|p_{\phi}\right|=$ $\sqrt{p^{2}+m^{2}-\lambda \phi}$ decreases toward zero. By convention, we then choose the negative sign for $p_{\phi}$ (the upper sign in (10) ), and $q(\phi)$ is increasing for positive $p$. In the other branch, with $\phi$ decreasing at values smaller than $\phi_{\mathrm{t}}$, the square root increases and $q(\phi)$ is growing if the opposite sign of $p_{\phi}$ is used. However, choosing the positive sign for $p_{\phi}$ (the lower sign in (10) ) should also mean that the original trajectory is followed in reverse. This is the general problem of the lack of a clear distinction between different branches of forward and backward evolution before as well as after the turning point, mentioned in the beginning of this section. We have to separate the choice of the sign of $\mathrm{d} \phi / \mathrm{d} \epsilon$ from the choice of the sign of $p_{\phi}$, even though they are linked by $\mathrm{d} \phi / \mathrm{d} \epsilon=2 p_{\phi}$ in classical equations of motion. Such a separation turns out to be possible in path-integral treatments.

Before we continue, it is useful to put our considerations in a more general context, as realized for instance in models of general relativity. In such cases, $\epsilon$ would be considered as proper time, the physical time parameter used by observers. The success of any quantum prescription for evolution should therefore be judged by comparison with $\epsilon$-evolution. However, a parameter such as $\epsilon$ does not appear in canonically quantized theories, thus

motivating the use of an internal time. If there is a monotonic relationship $\phi(\epsilon)$ for a global internal time, $\phi$ and $\epsilon$ can be used interchangeably without problems. If not, we have to find a way of describing evolution in terms of $\phi$ in spite of its turning around. Such 
a description should be able to include all branches of the classical $\epsilon$-evolution in which semiclassical behavior is expected to be possible. (For instance, both expansion and collapse should be realizable semiclassically in the internal-time formulation of a recollapsing cosmological model.) Taking our model as an example, we should be able to construct quantum evolution with a monotonic $\langle\hat{q}\rangle(\tau)$ even across a turning point of internal time, in correspondence with the monotonic $q(\epsilon)$ implied by (8) .

In order to solve this problem, we disentangle the branches by writing $\phi$-evolution globally with a monotonic dependence on a time parameter $\tau$. We introduce this new parameter so that $\phi=\tau$ if $\lambda \tau<p_{\mathrm{t}}^{2}+m^{2}$ (before the turning point as measured by $\tau$ ) and

$$
\phi=2 \phi_{\mathrm{t}}-\tau=2 \lambda^{-1}\left(p_{\mathrm{t}}^{2}+m^{2}\right)-\tau
$$

if $\lambda \tau>p_{\mathrm{t}}^{2}+m^{2}$. The two ranges of $\tau$ correspond to the two branches of $\phi$-evolution towards the turning point at $\phi_{\mathrm{t}}$ and away from it. The parameter $\tau$ defined in this way provides a continuous and monotonic parameterization of the whole trajectory of $\phi$. In particular, $q(\tau)$ is a monotonic function, in contrast to $q(\phi)$. The extended range of $\tau$ ensures that we have now a clear distinction of different phases, $p_{\phi}$ negative or positive for forward and backward evolution, before the turning point if $\tau<\phi_{\mathrm{t}}$ and after if $\tau>\phi_{\mathrm{t}}$. (At this point, a comparison with topological turning points is useful: Even though the treatment of signs of $p_{\phi}$ is then more straightforward, the extension of the time parameter given by (11) is similar to the unwinding of circular internal times constructed in [17].)

The "time reflection" introduced in the parameterization (11) has implications for the form of the $\phi$-Hamiltonian, governing evolution of $(q, p)$ with respect to internal time $\phi$. In systems in which $\phi$ is a global internal time, this Hamiltonian is just $p_{\phi}(q, p)$ obtained by solving the constraint $C=0$ for $p_{\phi}$. In our model with a $\phi$-dependent potential, the $\phi$-Hamiltonian is time dependent. For evolution through the turning point, we should write this Hamiltonian in terms of $\tau$ instead of $\phi$. Before the turning point we just replace $\phi$ in $p_{\phi}=-\sqrt{p^{2}+m^{2}-\lambda \phi}$ with $\tau$, so that $H_{\tau}(q, p)=-\sqrt{p^{2}+m^{2}-\lambda \tau}$ if $\tau<\phi_{\mathrm{t}}$. After the turning point, we replace $\phi$ by $\tau$ using (11), and then have

$$
H_{\tau}(q, p)=-\sqrt{\lambda \tau-m^{2}-\left(2 p_{\mathrm{t}}-p^{2}\right)}=-\sqrt{\lambda \tau-m^{2}-p^{2}}
$$

using the conservation of $p=p_{\mathrm{t}}$. The Hamiltonian

$$
H_{\tau}(q, p)=-\sqrt{\left|p^{2}+m^{2}-\lambda \tau\right|}= \begin{cases}-\sqrt{p^{2}+m^{2}-\lambda \tau} & \text { if } \tau<\phi_{\mathrm{t}} \\ -\sqrt{\lambda \tau-m^{2}-p^{2}} & \text { if } \tau>\phi_{\mathrm{t}}\end{cases}
$$

generates the equation of motion

$$
\frac{\mathrm{d} q}{\mathrm{~d} \tau}=\left\{q, H_{\tau}\right\}=-\operatorname{sgn}\left(p^{2}+m^{2}-\lambda \tau\right) \frac{p}{\sqrt{\left|p^{2}+m^{2}-\lambda \tau\right|}} .
$$

Even though we use the negative sign in solving for $p_{\phi}$ before and after the turning point, the time reflection contained in $\phi(\tau)$ implies that $q(\tau)$ after the turning point is described by backward evolution, In order to have forward evolution, we should multiply the $\tau$ Hamiltonian with $\operatorname{sgn}\left(p^{2}+m^{2}-\lambda \tau\right)$. In a path-integral treatment, to which we turn now, this factor is provided automatically because the Hamiltonian for the evolution of $(q, p)$ is derived from the term $\dot{\phi} p_{\phi}$ in the action, not just $p_{\phi}$. 


\subsection{Path-integral formulation}

We can transfer our classical parameterization to the path-integral quantization if we change the original gauge-fixing condition for a global internal time to

$$
\phi-\tau \theta\left(p_{\mathrm{t}}^{2}+m^{2}-\lambda \tau\right)-\left(\frac{2}{\lambda}\left(p_{\mathrm{t}}^{2}+m^{2}\right)-\tau\right) \theta\left(\lambda \tau-p_{\mathrm{t}}^{2}-m^{2}\right)=0,
$$

suitable for a local internal time with a single turning point. Path-integrating over $\phi$ and $p_{\phi}$ solves the constraint and the gauge-fixing condition, so that the $\phi$-dependence of the action is turned into

$$
\dot{\phi} p_{\phi}=-\sqrt{p^{2}+m^{2}-\lambda \tau} \theta\left(p_{\mathrm{t}}^{2}+m^{2}-\lambda \tau\right)+\sqrt{\lambda \tau-m^{2}-\left(2 p_{\mathrm{t}}^{2}-p^{2}\right)} \theta\left(\lambda \tau-p_{\mathrm{t}}^{2}-m^{2}\right) .
$$

(Taking a $\tau$-derivative of the step functions in (15) contributes two delta functions, which however cancel out.) In the specific model, $p=p_{\mathrm{t}}$ is conserved, so that the result can simply be written as

$$
\dot{\phi} p_{\phi}=-\operatorname{sgn}\left(p^{2}+m^{2}-\lambda \tau\right) \sqrt{\left|p^{2}+m^{2}-\lambda \tau\right|} .
$$

The Hamiltonian is therefore always real, but its sign changes according to the branch of $\phi$-evolution (without changing the sign of $p_{\phi}$ ).

In this simple example with $q$-independent potential, the path-integral can be computed in the momentum representation. We will first assume that only ranges of evolution are considered which do not contain a turning point. Using the general solution

$$
\psi(p, \phi)=c(p) \exp \left(2 i(3 \lambda \hbar)^{-1}\left(p^{2}+m^{2}-\lambda \phi\right)^{3 / 2}\right)
$$

of the Schrödinger equation

$$
i \hbar \frac{\partial \psi}{\partial \phi}=\sqrt{p^{2}+m^{2}-\lambda \phi} \psi
$$

obtained after quantizing the deparameterized constraint, it is easier to compute the propagator directly instead of integrating over paths. Choosing a complete set of functions

$$
c_{x}(p)=\frac{1}{\sqrt{2 \pi \hbar}} e^{i x p / \hbar}
$$

the propagator is given by

$$
\begin{aligned}
\left(p_{\mathrm{b}} \tau_{\mathrm{b}} \mid p_{\mathrm{a}} \tau_{\mathrm{a}}\right) & =\int \mathrm{d} x \psi_{x}\left(p_{\mathrm{b}}, \tau_{\mathrm{b}}\right) \psi_{x}\left(p_{\mathrm{a}}, \tau_{\mathrm{a}}\right)^{*} \\
& =\delta\left(p_{\mathrm{b}}-p_{\mathrm{a}}\right) \exp \left(-2 i(3 \lambda \hbar)^{-1}\left(\left(p_{\mathrm{b}}^{2}+m^{2}-\lambda \tau_{\mathrm{b}}\right)^{3 / 2}-\left(p_{\mathrm{a}}^{2}+m^{2}-\lambda \tau_{\mathrm{a}}\right)^{3 / 2}\right)\right) .
\end{aligned}
$$

The assumption that no turning point be contained in the range $\left(\tau_{\mathrm{a}}, \tau_{\mathrm{b}}\right)$ can be fulfilled only if the wave function is not supported on momenta $p$ for which $p^{2}+m^{2}-\lambda \tau$ is negative 
for $\tau$ in the given range. Any Gaussian clearly violates this assumption, so that we have to be more careful with turning points even if we are interested in semiclassical evolution close to a piece of a classical trajectory that stays away from the turning point. We can, however, combine the specific result (21) with (17) and the general composition rule (5), written in the momentum representation, in order to compute the complete propagator for arbitrary initial states. We write

$$
\begin{aligned}
\left(p_{\mathrm{b}} \tau_{\mathrm{b}} \mid p_{\mathrm{a}} \tau_{\mathrm{a}}\right)= & \int \mathrm{d} p_{\mathrm{t}}\left(p_{\mathrm{b}} \tau_{\mathrm{b}} \mid p_{\mathrm{t}} \tau_{\mathrm{t}}\right)\left(p_{\mathrm{t}} \tau_{\mathrm{t}} \mid p_{\mathrm{a}} \tau_{\mathrm{a}}\right) \\
= & \int \mathrm{d} p_{\mathrm{t}}\left(\int D q D p \exp \left(\frac{i}{\hbar} \int_{\tau_{\mathrm{t}}}^{\tau_{\mathrm{b}}}\left(\dot{q} p+\sqrt{\lambda \tau-m^{2}-\left(2 p_{\mathrm{t}}^{2}-p^{2}\right)}\right)\right)\right) \\
& \times\left(\int D q D p \exp \left(\frac{i}{\hbar} \int_{\tau_{\mathrm{a}}}^{\tau_{\mathrm{t}}}\left(\dot{q} p-\sqrt{p^{2}+m^{2}-\lambda \tau}\right)\right)\right) \\
= & \int \mathrm{d} p_{\mathrm{t}} \delta\left(p_{\mathrm{b}}-p_{\mathrm{t}}\right) \exp \left(2 i(3 \lambda \hbar)^{-1}\left(\lambda \tau_{\mathrm{b}}-m^{2}-\left(2 p_{\mathrm{t}}^{2}-p_{\mathrm{b}}^{2}\right)\right)^{3 / 2}\right) \\
& \times \delta\left(p_{\mathrm{t}}-p_{\mathrm{a}}\right) \exp \left(-2 i(3 \lambda \hbar)^{-1}\left(p_{\mathrm{a}}^{2}+m^{2}-\lambda \tau_{\mathrm{a}}\right)^{3 / 2}\right) \\
= & \delta\left(p_{\mathrm{b}}-p_{\mathrm{a}}\right) \exp \left(2 i(3 \lambda \hbar)^{-1}\left(\left(\lambda \tau_{\mathrm{b}}-m^{2}-p_{\mathrm{a}}^{2}\right)^{3 / 2}-\left(p_{\mathrm{a}}^{2}+m^{2}-\lambda \tau_{\mathrm{a}}\right)^{3 / 2}\right)\right)
\end{aligned}
$$

No divergences related to the turning point are present.

We can now use the propagator to evolve an initial state "through the turning point." (Strictly speaking, such a state never evolves completely through the turning point since it is supported on momenta $p$ for which, at any finite $\tau, \phi$ has not yet reached its turning point.) We assume an initial Gaussian state

$$
\psi\left(p_{\mathrm{a}}, \tau_{\mathrm{a}}\right)=\frac{1}{(2 \pi)^{1 / 4} \sqrt{\sigma}} \exp \left(-\frac{\left(p_{\mathrm{a}}-p_{0}\right)^{2}}{4 \sigma^{2}}-\frac{i}{\hbar} q_{0} p_{\mathrm{a}}\right) .
$$

It is easy to compute

$$
\begin{aligned}
\psi\left(p_{\mathrm{b}}, \tau_{\mathrm{b}}\right)= & \int \mathrm{d} p_{\mathrm{a}}\left(p_{\mathrm{b}} \tau_{\mathrm{b}} \mid p_{\mathrm{a}} \tau_{\mathrm{a}}\right) \psi\left(p_{\mathrm{a}}, \tau_{\mathrm{a}}\right) \\
= & \frac{1}{(2 \pi)^{1 / 4} \sqrt{\sigma}} \exp \left(-\frac{\left(p_{\mathrm{b}}-p_{0}\right)^{2}}{4 \sigma^{2}}-\frac{i}{\hbar} q_{0} p_{\mathrm{b}}\right) \\
& \times \exp \left(2 i(3 \lambda \hbar)^{-1}\left(\left|p_{\mathrm{b}}^{2}+m^{2}-\lambda \tau_{\mathrm{b}}\right|^{3 / 2}-\left|p_{\mathrm{b}}^{2}+m^{2}-\lambda \tau_{\mathrm{a}}\right|^{3 / 2}\right)\right) .
\end{aligned}
$$

The time-dependent expectation value of $\hat{q}$ in this state is given by

$$
\langle\hat{q}\rangle(\tau) \sim q_{0}-\frac{2}{\lambda}\left\langle\hat{p}\left(\sqrt{\hat{p}^{2}+m^{2}-\lambda \tau}-\sqrt{\hat{p}^{2}+m^{2}-\lambda \tau_{\mathrm{a}}}\right)\right\rangle
$$

if $\tau$ and $\tau_{\mathrm{a}}$ are well before the classical turning point belonging to $p_{0}$, and

$$
\langle\hat{q}\rangle(\tau) \sim q_{0}+\frac{2}{\lambda}\left\langle\hat{p}\left(\sqrt{\lambda \tau-\hat{p}^{2}-m^{2}}+\sqrt{\hat{p}^{2}+m^{2}-\lambda \tau_{\mathrm{a}}}\right)\right\rangle
$$


if $\tau_{\mathrm{a}}$ is before and $\tau$ well after the turning point belonging to $p_{0}$. (For $\tau$ not too close to the $p$ dependent turning point $\tau_{\mathrm{t}}$, the spread of the Gaussian (24) to values of $p$ for which $\tau<\tau_{\mathrm{t}}$ can be ignored in an approximation.) As required for the classical behavior, discussed after Eq. (10), $\langle\hat{q}\rangle(\tau)$ increases monotonically. Moreover, for a state sharply peaked in the momentum, the expectation value in (26) is well approximated by the classical limit (10), both before and after the turning point. More precisely, for such a state one can approximate

$$
\begin{aligned}
\left\langle\hat{p} \sqrt{\lambda \tau-\hat{p}^{2}-m^{2}}\right\rangle & \approx\langle\hat{p}\rangle \sqrt{\lambda \tau-\langle\hat{p}\rangle^{2}-m^{2}}+\frac{\mathrm{d}^{2}\left(\langle\hat{p}\rangle \sqrt{\lambda \tau-\langle\hat{p}\rangle^{2}-m^{2}}\right)}{\mathrm{d}\langle\hat{p}\rangle^{2}}(\Delta p)^{2} \\
& =\langle\hat{p}\rangle \sqrt{\lambda \tau-\langle\hat{p}\rangle^{2}-m^{2}}\left(1-\left(3-\frac{\langle\hat{p}\rangle^{2}}{\lambda \tau-\langle\hat{p}\rangle^{2}-m^{2}}\right) \frac{(\Delta p)^{2}}{\lambda \tau-\langle\hat{p}\rangle^{2}-m^{2}}\right)
\end{aligned}
$$

up to higher moments of $p$. (We are using methods of canonical effective equations, following [18, 19].) Even if the state remains starply peaked in $p$ during evolution as the turning point is approached, the semiclassical approximation to (26) eventually becomes invalid close to the turning point because of inverse powers of $\lambda \tau-\langle\hat{p}\rangle^{2}-m^{2} \approx\left\langle\hat{p}_{\phi}\right\rangle \approx 0$. This result is in agreement with canonical effective treatments, which we will compare with in the next section. But first, we briefly summarize general lessons that can be drawn from our simple example.

\subsection{A formal definition of evolution through a turning point}

It is not straightforward to provide a good definition of evolution through a turning point. As our example suggests, it is important to distinguish between local relational evolution with respect to some internal time $\phi$, and globally defined evolution with respect to a time parameter $\tau$. The latter should locally agree with the former (or its reverse) in small intervals not including a turning point, so that it can be seen as patched-up evolution obtained by matching different ranges of local evolution. Instead of unitary evolution with respect to $\phi$, as required in the case of global internal times, we can then impose two minimal conditions for meaningful evolution through a turning point:

1. We have the composition law (5) for transition amplitudes.

2. There should be semiclassical states well before and well after the turning point, whose quantum evolution is well approximated by the classical evolution.

The first condition is a replacement of unitary evolution operators. The second one is necessary because previous attempts to obtain evolution through a turning point, based on deparameterization or the proposal of [20], have led to a "freezing" of evolution after a turning point [21, 22] which does not agree with classical evolution. No freezing happens in our formalism, as seen in equation (26).

In our specific formulation of the second condition, we do not require that a single state which is semiclassical before the turning point always evolves to a semiclassical state after 
the turning point. However, using the general transition amplitudes, it should be possible to follow an initial state for a brief interval before or after the turning point, and obtain nearly classical evolution for an appropriate choice of semiclassical state. This property allows one to verify that different branches before and after the turning point are indeed matched correctly, as we have seen in the monotonic behavior of $\langle\hat{q}\rangle(\tau)$ in our example. (This condition may have to be relaxed if there are intervals in which turning points are reached in rapid succession. Such a behavior may leave no time to set up semiclassical evolution [23]. We could simply exclude such ranges from the condition and consider the succession of turning points as a single transition phase, on both sides of which semiclassical behavior should be possible.)

\section{Comparison with canonical treatment}

It is difficult to reconcile the requirement of unitary evolution of wave functions in a Hilbert space with the local nature of generic internal times. We have arrived at an alternative of the canonical requirement of a self-adjoint Hamiltonian or unitary evolution operator in the preceding section. A consistent canonical formulation of local internal times can also be obtained, at least semiclassically [13, 14, if one describes states not by wave functions in a Hilbert space but as algebraic expectation-value functionals. A state is then a positive linear functional mapping the algebra of observables to the complex numbers. Conditions on operators on wave functions in a Hilbert space are not as apparent in such a formulation and may therefore be relaxed, although the price at which such a generalization would be done is difficult to estimate at the semiclassical level. Nevertheless, a comparison with the new results of a path-integral formulation is of interest.

For constrained systems, there are two kinds of algebras of observables, the kinematical one and the physical one. As shown by the treatment of effective constraints, the constraints themselves as well as reality conditions can be imposed by referring solely to expectation-value functionals and their kinematical algebraic relationships, deriving in this way properties of physical states without having to construct a physical Hilbert space with an explicit inner product. The latter has so far been found only for systems with global internal times, following [24], so that it presents one of the major obstacles toward a solution of the problem of time in canonical terms. Also the problem of finding quantum observables is often a difficult one. The algebraic methods of [13, 14] map the quantum problem to an analogous one of classical type, formulated on an enlarged phase space. Classical methods and approximations can then be used to compute expectation values and moments of quantum observables, without the need to find operators for observables or a physical Hilbert space.

Canonical effective constraints are usually formulated for expectation-value functionals parameterized by the expectation values and moments assigned by a state to a set of basic variables. Using canonical basic operators $\hat{q}$ and $\hat{p}$, the variables are then $q:=\langle\hat{q}\rangle$ and $p:=\langle\hat{p}\rangle$ as well as

$$
\Delta\left(q^{a} p^{b}\right):=\left\langle(\hat{q}-q)^{a}(\hat{p}-p)^{b}\right\rangle_{\mathrm{Weyl}}
$$


in totally symmetric or Weyl ordering. (For the sake of uniform notation, we slightly modify the usual denotation of fluctuations by identifying $\Delta\left(q^{2}\right)=(\Delta q)^{2}$.) A generalization to several canonical degrees of freedom is straightforward. These variables form a phase space with Poisson brackets derived from the commutator in the algebra, extending

$$
\{\langle\hat{A}\rangle,\langle\hat{B}\rangle\}:=\frac{\langle[\hat{A}, \hat{B}]\rangle}{i \hbar}
$$

to moments by the Leibniz rule. The Hamiltonian flow generated by a function on the space of states, with basic expectation values and moments as coordinates, is then equivalent to the Schrödinger flow of the expectation-value functional [18].

Using the notation of our model system, $(q, p)$ along with $\left(\phi, p_{\phi}\right)$ are kinematical variables. They are subject to a constraint $C=0$ and its gauge flow. After quantization, physical states are those annihilated by the constraint operator: $\hat{C} \psi=0$. For expectationvalue functionals, this means that their basic expectation values and moments are not arbitrary but subject to several independent conditions

$$
\langle\hat{C}\rangle=0 \quad, \quad\langle(\hat{q}-q) \hat{C}\rangle=0 \quad, \quad\langle(\hat{p}-p) \hat{C}\rangle=0 \quad, \quad\langle(\hat{\phi}-\phi) \hat{C}\rangle=0 \quad, \quad\left\langle\left(\hat{p}_{\phi}-p_{\phi}\right) \hat{C}\right\rangle=0
$$

and so on with more factors of the basic kinematical variables on the left of $\hat{C}$. (It is important to fix the ordering of $\hat{C}$ either to the left or the right, so that a first-class constrained system is obtained [12.) All these conditions can be expressed as constraint functions on expectation values and moments by using expansions such as

$$
\langle C(\hat{q}, \hat{p})\rangle=\langle C(q+(\hat{q}-q), p+(\hat{p}-p))\rangle=C(q, p)+\sum_{a, b} \frac{1}{a ! b !} \frac{\partial^{a+b} C(q, p)}{\partial^{a} q \partial^{b} p} \Delta\left(q^{a} p^{b}\right)
$$

and again straightforward extensions to several independent canonical pairs. (The expansion is formal unless $\hat{C}$ is polynomial in basic operators.) For our model constraint $C=p_{\phi}^{2}-H(q, p, \phi)^{2}$, we obtain

$$
\begin{aligned}
\langle\hat{C}\rangle= & p_{\phi}^{2}-H(q, p, \phi)^{2}+\Delta\left(p_{\phi}^{2}\right) \\
& -\left(\frac{\partial^{2} H^{2}}{\partial q^{2}} \Delta\left(q^{2}\right)+\frac{\partial^{2} H^{2}}{\partial p^{2}} \Delta\left(p^{2}\right)+\frac{\partial^{2} H^{2}}{\partial \phi^{2}} \Delta\left(\phi^{2}\right)\right. \\
& \left.+2 \frac{\partial^{2} H^{2}}{\partial q \partial p} \Delta(q p)+2 \frac{\partial^{2} H^{2}}{\partial q \partial \phi} \Delta(q \phi)+2 \frac{\partial^{2} H^{2}}{\partial p \partial \phi} \Delta(p \phi)\right) \\
\langle(\hat{q}-q) \hat{C}\rangle= & 2 p_{\phi} \Delta\left(q p_{\phi}\right)-2 H(q, p, \phi)\left(\frac{\partial H}{\partial q} \Delta\left(q^{2}\right)+\frac{\partial H}{\partial p} \Delta(q p)+\frac{\partial H}{\partial \phi} \Delta(q \phi)\right)
\end{aligned}
$$

and so on.

In our main example, we assume $H^{2}=p^{2}+m^{2}-\lambda \phi$, and have

$$
\langle\hat{C}\rangle=p_{\phi}^{2}-p^{2}-m^{2}+\lambda \phi+\Delta\left(p_{\phi}^{2}\right)-\Delta\left(p^{2}\right) .
$$


Since we have expressed the quantum constrained system as a Hamiltonian one of classical type, we can try to follow classical deparameterization techniques as much as possible. Choosing $\phi$ as (local) internal time as before, we should solve the constraint equation $\langle\hat{C}\rangle=0$ for the momentum $p_{\phi}$. However, the quantum nature of our system implies that not only expectation values $\phi$ and $p_{\phi}$ appear in the constraints, but also moments such as $\Delta\left(p_{\phi}^{2}\right)$ in (34). Solving (34) for $p_{\phi}$ then does not give us a Hamiltonian for $\phi$-evolution of the pair $(q, p)$ and their moments, unless we find a way to compute $\Delta\left(p_{\phi}^{2}\right)$ independently.

This task is made possible by the presence of higher-order constraints in our system (30). In particular, the constraint

$$
\left\langle\left(\hat{p}_{\phi}-p_{\phi}\right) \hat{C}\right\rangle=2 p_{\phi} \Delta\left(p_{\phi}^{2}\right)-2 p \Delta\left(p p_{\phi}\right)+\lambda\left(\Delta\left(\phi p_{\phi}\right)-\frac{1}{2} i \hbar\right)=0
$$

provides a condition on $\Delta\left(p_{\phi}^{2}\right)$ independent of (34). (There is an imaginary term in (35) because we are ordering $\hat{C}$ to the right in (30), while moments such as $\Delta\left(\phi p_{\phi}\right)$ are ordered totally symmetrically. Effective constraints are therefore complex-valued in terms of kinematical variables. Reality conditions will be imposed below after solving the constraints and eliminating their gauge flow, thereby transitioning to physical states.)

Solving (35) for $\Delta\left(p_{\phi}^{2}\right)$ still does not give us a solution independent of $\left(\phi, p_{\phi}\right)$-moments because there are contributions from $\Delta\left(p p_{\phi}\right)$ and $\Delta\left(\phi p_{\phi}\right)$ in (35). We find an equation for $\Delta\left(p p_{\phi}\right)$ if we compute

$$
\langle(\hat{p}-p) \hat{C}\rangle=2 p_{\phi} \Delta\left(p p_{\phi}\right)-2 p \Delta\left(p^{2}\right)+\lambda \Delta(\phi p),
$$

and one for $\Delta\left(\phi p_{\phi}\right)$ given by

$$
\langle(\hat{\phi}-\phi) \hat{C}\rangle=2 p_{\phi} \Delta\left(\phi p_{\phi}\right)+i \hbar p_{\phi}-2 p \Delta(\phi p)+\lambda \Delta\left(\phi^{2}\right) .
$$

The $\left(\phi, p_{\phi}\right)$-moments seem to proliferate, but most of them are subject to gauge flows generated by the constraints (30). They can therefore be eliminated when the gauge is fixed or factored out. As shown in [13, 14], a good partial gauge fixing of the flow generated by the constraints (35), (36), (37) and $\langle(\hat{q}-q) \hat{C}\rangle$ (which latter we will not be using in explicit form) is given by the conditions

$$
\Delta\left(\phi^{2}\right)=\Delta(\phi q)=\Delta(\phi p)=0
$$

on moments of local internal time $\phi$, or the Zeitgeist according to [13, 14]. (Three conditions suffice to fix the four first-order constraints because the second-order moments do not form a symplectic phase space, while (29) defines a non-invertible Poisson bracket.) These gauge-fixing conditions implement the expectation that choosing $\phi$ as internal time should turn $\phi$ into a time parameter of classical type, which does not have non-trivial fluctuations or correlations.

The gauge-fixing conditions (38) do not contain $\Delta\left(\phi p_{\phi}\right)$, which instead is determined by effective constraints. Using the gauge-fixing conditions, we can solve (37) for

$$
\Delta\left(\phi p_{\phi}\right)=-\frac{1}{2} i \hbar
$$


(36) for

$$
\Delta\left(p p_{\phi}\right)=\frac{p}{p_{\phi}} \Delta\left(p^{2}\right),
$$

and (35) for

$$
\Delta\left(p_{\phi}^{2}\right)=\frac{p}{p_{\phi}} \Delta\left(p p_{\phi}\right)+\frac{i \hbar \lambda}{2 p_{\phi}}=\frac{p^{2}}{p_{\phi}^{2}} \Delta\left(p^{2}\right)+\frac{i \hbar \lambda}{2 p_{\phi}} .
$$

(Note that the imaginary value (39) ensures that the uncertainty relation for $\left(\phi, p_{\phi}\right)$ is formally obeyed even though $\Delta\left(\phi^{2}\right)=0$ according to (38).) We finally obtain the $\phi$ Hamiltonian for the evolution of $(q, p)$ and their moments by solving

$$
\langle\hat{C}\rangle=p_{\phi}^{2}-p^{2}-m^{2}+\frac{p^{2}-p_{\phi}^{2}}{p_{\phi}^{2}} \Delta\left(p^{2}\right)+\lambda \phi+\frac{1}{2} i \hbar \frac{\lambda}{p_{\phi}}=0 .
$$

At this point, we have to select appropriate reality conditions. After solving the constraints, the non-time variables $(q(\phi), p(\phi))$ and their moments should become physical observables; they should therefore be real. Moreover, $p_{\phi}$ will be used as the Hamiltonian generating the evolution of $(q, p)$ and their moments with respect to $\phi$, and therefore should be real too. The remaining variables in (42) cannot all be real because of the imaginary term. The only consistent choice is to allow $\phi$ (defined so far as the kinematical expectation value of $\hat{\phi}$ ) to take complex values, since time is not an observable after deparameterization. The imaginary part $\operatorname{Im}\langle\hat{C}\rangle=0$ of (42) then implies the imaginary part

$$
\operatorname{Im} \phi=-\frac{\hbar}{2 p_{\phi}}
$$

of time, while Re $\phi$ remains unconstrained and free to play the role of an evolution parameter. The real part of (42) is a quadratic equation for $p_{\phi}^{2}$, giving the $\phi$-Hamiltonian

$$
p_{\phi}= \pm \sqrt{\frac{1}{2}\left(p^{2}+m^{2}-\lambda \operatorname{Re} \phi+\Delta\left(p^{2}\right) \pm \sqrt{\left(p^{2}+m^{2}-\lambda \operatorname{Re} \phi+\Delta\left(p^{2}\right)\right)^{2}-4 p^{2} \Delta\left(p^{2}\right)}\right)} .
$$

We choose the positive sign for the interior square root, ensuring that we have the classical Hamiltonian for small $\Delta\left(p^{2}\right)$. The remaining sign choice is then the usual one, distinguishing forward evolution from backward evolution.

The Hamiltonian (44) is non-linear in the moment $\Delta\left(p^{2}\right)$. Since we derived it from constraints expanded up to leading order in moments, we should expand the square roots in (44) to the same order:

$$
\pm p_{\phi}=\sqrt{p^{2}+m^{2}-\lambda \operatorname{Re} \phi}+\frac{1}{2} \frac{m^{2}-\lambda \operatorname{Re} \phi}{\left(p^{2}+m^{2}-\lambda \operatorname{Re} \phi\right)^{3 / 2}} \Delta\left(p^{2}\right)=H(p, \phi)+\frac{1}{2} \frac{\partial^{2} H}{\partial^{2} p} \Delta\left(p^{2}\right) .
$$

The final expression is just the expansion of the deparameterized effective Hamiltonian $\langle H(\hat{p}, \phi)\rangle$ as in (31) in which $\phi$ is treated as internal time rather than an operator. This result agrees with the path-integral expression (4) obtained after gauge fixing. 
One can solve for some of the moments as well. For fluctuations of $p_{\phi}$, for instance, we obtain

$$
\Delta\left(p_{\phi}^{2}\right)=\frac{p^{2}}{p^{2}+m^{2}-\lambda \operatorname{Re} \phi} \Delta\left(p^{2}\right)+\frac{1}{2} i \hbar \frac{\lambda}{\sqrt{p^{2}+m^{2}-\lambda \operatorname{Re} \phi}}
$$

which is not real. The expectation value of $\hat{p}_{\phi}^{2}$ is then

$$
\left\langle\hat{p}_{\phi}^{2}\right\rangle=\Delta\left(p_{\phi}^{2}\right)+p_{\phi}^{2}=p^{2}+m^{2}+\Delta\left(p^{2}\right)-\lambda\left(\operatorname{Re} \phi-\frac{1}{2} i \hbar \frac{1}{\sqrt{p^{2}+m^{2}-\lambda \phi}}\right) .
$$

This is the correct result obtained from $\langle\hat{C}\rangle=0$, taking into account an imaginary part of $\langle\hat{\phi}\rangle$ (and treating $\hbar \Delta\left(p^{2}\right)$ as a higher-order term).

If we could use a physical Hilbert space while treating $\phi$ as an internal time, only $\hat{q}$ and $\hat{p}$ would be well-defined among the basic operators. While expectation values and moments of $\left(\phi, p_{\phi}\right)$ exist on the kinematical Hilbert space and are real, they do not exist as independent variables on the physical Hilbert space, and they need not be subject to reality conditions. Only the evolution generator in internal time, given by $\left\langle\hat{p}_{\phi}\right\rangle$ as a function of $(q, p)$-moments, must be real as used above. The resulting imaginary part of the kinematical $\langle\hat{\phi}\rangle$ turns out to be an important property of solutions to effective constraints, because it allows one to change local internal times by gauge transformations. If one transforms from internal time $\phi$ to internal time $q$, for instance, the imaginary part of $\phi$ is removed and replaced by an imaginary part of the new time $q$. (For details on this result, which are rather technical, we refer to [13, 14].)

If transformations between local internal times can be implemented at the level of path integrals, an imaginary part of the time expectation value would seem to be an important property as well. However, after gauge fixing, it is no longer possible to compute a time expectation value. In fact, the path integral of a constrained system, which contains a factor of $\delta(C)$ in its integrand, amounts to a projector on the physical Hilbert space on which $\phi$ does not exist as an operator. It is conceivable that an imaginary contribution to time appears in two possible ways: (i) One could impose a gauge fixing which mixes different time choices, such as $G=\epsilon q+(1-\epsilon) \phi-\tau=0$ for fixed $\tau$, with a transition of $\epsilon$ from zero to one switching time. With such a choice, factors from the FaddeevPopov determinant and the delta function $\delta(C)$ no longer cancel out, so that the relevant path integrals are more complicated. (ii) A careful discretization of paths in the unfixed integral with a time-dependent constraint could show subtleties that require an imaginary contribution. We will not pursue these rather technical issues here, but note the main result of this section: A comparison of evolution (45) in the canonical effective treatment (to leading semiclassical order) agrees with what one would expect from the path-integral formula (4), before and after a turning point. 


\section{$5 \quad$ Implications}

In physical terms, the problem of time has two main aspects, both related to the choice of internal time variables for relational evolution. For the most part, we have addressed the question of how one can define evolution through a turning point of a local internal time, and only briefly commented on the possibility of changing the choice of time. The former aspect is important for a complete definition of relational evolution, while the latter is crucial if one tries to ensure covariance of the quantum system in the sense that it provides predictions independent of the choice of internal time. Our path-integral treatment has, so far, not led to new results on the question of transforming between different internal times, for which kinematical aspects and non-real time expectation values seem to play a role according to the canonical treatment of [13, 14].

Regarding evolution through a turning point of a local internal time, we have provided a specific definition based on an example solved explicitly. The path-integral treatment suggests several simplifications compared with the canonical one. Solutions of effective constraints lead to quantities which are singular at the turning point where $p_{\phi}=0$, seen for instance in the imaginary part of time (43) or in semiclassical corrections to the $\phi$ Hamiltonian in (45). However, the imaginary part of time is a kinematical quantity and therefore auxiliary, and the contribution of (45) singular at $p_{\phi}=0$ has been obtained from an expansion of (44) which does not appear to be singular at the turning point. The effective treatment, to orders considered so far, cannot tell whether the singularity in (45) is only apparent or real. In [13, 14, turning points of internal times therefore had to be evaded by transforming to a different internal time before a turning point is reached. While such transformations are possible in the effective treatment, they can, as pointed out in [23], lead to problems in regimes in which turning points of different variables are close to one another, such as chaotic systems. Such transformations may be difficult to define at the path-integral level, but our results here make one promising suggestion: Turning points appear to be much less singular in this formalism, so that we can evolve right up to the turning point from one side and, after a "reflection" (11) of time, continue onwards. The evolved state then indeed suggests that a semiclassical treatment should lead to difficulties near a turning point of local internal time, as seen explicitly in the expansion of $\langle\hat{q}\rangle(\tau)$ by moments in our model: There are not only terms of the standard semiclassical form $\Delta\left(p^{2}\right) / p^{2}$, which can always be chosen small for a suitable semiclassical state, but also $\Delta\left(p^{2}\right) / p_{\phi}^{2}$, which are large near $p_{\phi}=0$ for any state. Without the moment expansion, however, we have achieved a direct matching of branches before and after the turning point, without any divergence or freezing (as in [21, 22]) of the evolution. Such

a direct matching might eliminate problems encountered in semiclassical treatments of chaotic systems, pointed out in [23].

In our model system, a well-defined quantum evolution through a turning point of internal time has been provided, but the proposal is not free of potential practical problems in general. For instance, the reflection (11) refers to the values which the evolving variables take at the turning point. In our simple example, this expression depends only on the momentum $p$ which is a constant of motion. The value $p_{\mathrm{t}}$ at the turning point could 
therefore be identified with the momentum $p$ at any other time. With this substitution one obtains a $\phi$-Hamiltonian after the turning point which depends only on the evolving canonical variables $(q, p)$. More generally, there would be a complicated relation between $\left(q_{\mathrm{t}}, p_{\mathrm{t}}\right)$ and the canonical variables $(q, p)$ at some other time, or the $\phi$-Hamiltonian after a turning point would be non-local in time if $\left(q_{\mathrm{t}}, p_{\mathrm{t}}\right)$ are left without expressing them in terms of the evolving pair $(q, p)$. (Even the corresponding classical evolution governed by (12) would be non-local in $\phi$-time.)

Our analysis has led us to a new treatment of the sign of $p_{\phi}$ (the momentum of internal time) which differs from the usual choices for global internal times. In a path integral, the Hamiltonian for evolution of the non-time variables $(q, p)$ ends up being $\dot{\phi} p_{\phi}$ rather than just $p_{\phi}$. In order to disentangle forward and backward motion of a local internal time $\phi$ into a global time parameter $\tau$, we were led to choosing a gauge fixing (15) that includes a "time reflection" after the turning point. The time parameter $\tau$ can then continue running forward, even while the phase-space variable $\phi$ moves back after its turning point. The time reflection changes the sign of $\phi$, making it unnecessary to switch to a different sign of $p_{\phi}$. At this point, the analogy of the problem of time with general Gribov problems provides a justification for the new procedure, because the whole evolution can be formulated within one Gribov region. It is questionable whether this behavior can be modeled in a canonical treatment, where the Hamiltonian for $\phi$-evolution is $p_{\phi}$ rather than $\dot{\phi} p_{\phi}$. This difference between canonical and path-integral treatments may be the reason why it has been difficult to provide meaningful canonical evolutions through a turning point of local internal time.

\section{Acknowledgements}

We are grateful to Philipp Höhn for discussions. M. M. Amaral acknowledges support from CNPq, Brazil. This work was supported in part by NSF grant PHY-1307408.

\section{References}

[1] K. V. Kuchař, Time and interpretations of quantum gravity, In G. Kunstatter, D. E. Vincent, and J. G. Williams, editors, Proceedings of the 4 th Canadian Conference on General Relativity and Relativistic Astrophysics, Singapore, 1992. World Scientific

[2] C. J. Isham, Canonical quantum gravity and the problem of time, In Integrable systems, quantum groups, and quantum field theory, pages 157-287. Kluwer, Dordrecht, 1993

[3] E. Anderson, The Problem of Time in Quantum Gravity, In V. R. Frignanni, editor, Classical and Quantum Gravity: Theory, Analysis and Applications. Nova, New York, 2012, arXiv:1009.2157

[4] P. Malkiewicz, Reduced phase space approach to Kasner universe and the problem of time in quantum theory, Class. Quantum Grav. 29 (2012) 075008, arXiv:1105.6030 
[5] P. Malkiewicz, Multiple choices of time in quantum cosmology, Class. Quantum Grav. 32 (2015) 135004, arXiv:1407.3457]

[6] P. Malkiewicz, Clocks and dynamics in quantum models of gravity, arXiv:1601.04857]

[7] P. A. M. Dirac, Generalized Hamiltonian dynamics, Can. J. Math. 2 (1950) 129-148

[8] V. N. Gribov, Quantization of Nonabelian Gauge Theories, Nucl. Phys. B 139 (1978) $1-32$

[9] R. F. Sobreiro and S. P. Sorella, Introduction to the Gribov Ambiguities In Euclidean Yang-Mills Theories, hep-th/0504095

[10] J. B. Hartle and K. V. Kuchař, Path integrals in parametrized theories: The Free relativistic particle, Phys. Rev. D 34 (1986) 2323-2331

[11] H. Ikemori, Path Integral Representation of the Wave Function: The Relativistic Particle, Phys. Rev. D 40 (1989) 3512-3519

[12] M. Bojowald, B. Sandhöfer, A. Skirzewski, and A. Tsobanjan, Effective constraints for quantum systems, Rev. Math. Phys. 21 (2009) 111-154, arXiv:0804.3365]

[13] M. Bojowald, P. A. Höhn, and A. Tsobanjan, An effective approach to the problem of time, Class. Quantum Grav. 28 (2011) 035006, arXiv:1009.5953.

[14] M. Bojowald, P. A. Höhn, and A. Tsobanjan, An effective approach to the problem of time: general features and examples, Phys. Rev. D 83 (2011) 125023, arXiv:1011.3040,

[15] M. Henneaux and A. Slavnov, A note on the path integral for systems with primary and secondary second class constraints, Phys. Lett. B 338 (1994) 47-50

[16] M. Bojowald and A. Perez, Spin Foam Quantization and Anomalies, Gen. Rel. Grav. 42 (2010) 877, gr-qc/0303026

[17] B. Dittrich, P. A. Höhn, T. A. Koslowski, and M. I. Nelson, Chaos, Dirac observables and constraint quantization, arXiv:1508.01947]

[18] M. Bojowald and A. Skirzewski, Effective Equations of Motion for Quantum Systems, Rev. Math. Phys. 18 (2006) 713-745, math-ph/0511043

[19] M. Bojowald and A. Skirzewski, Quantum Gravity and Higher Curvature Actions, Int. J. Geom. Meth. Mod. Phys. 4 (2007) 25-52, [hep-th/0606232], Proceedings of "Current Mathematical Topics in Gravitation and Cosmology" (42nd Karpacz Winter School of Theoretical Physics), Ed. Borowiec, A. and Francaviglia, M.

[20] R. M. Wald, A Proposal for solving the 'problem of time' in canonical quantum gravity, Phys. Rev. D 48 (1993) 2377-2381, [gr-qc/9305024 
[21] A. Higuchi and R. M. Wald, Applications of a new proposal for solving the "problem of time' to some simple quantum cosmological models, Phys. Rev. D 51 (1995) 544-561, gr-qc/9407038

[22] M. Martín-Benito, G. A. Mena Marugán, and T. Pawlowski, Physical evolution in Loop Quantum Cosmology: The example of vacuum Bianchi I, Phys. Rev. D 80 (2009) 084038, arXiv:0906.3751

[23] P. A. Höhn, E. Kubalova, and A. Tsobanjan, Effective relational dynamics of a nonintegrable cosmological model, Phys. Rev. D 86 (2012) 065014, arXiv:1111.5193

[24] W. F. Blyth and C. J. Isham, Quantization of a Friedmann universe filled with a scalar field, Phys. Rev. D 11 (1975) 768-778 